\title{
Chimpanzees (Pan troglodytes) do not develop contingent reciprocity in an experimental task
}

\author{
Sarah Frances Brosnan · Joan B. Silk · Joseph Henrich • \\ Mary Catherine Mareno $\cdot$ Susan P. Lambeth · \\ Steven J. Schapiro
}

Received: 9 December 2008 / Revised: 4 February 2009 / Accepted: 9 February 2009 / Published online: 4 March 2009

(C) The Author(s) 2009. This article is published with open access at Springerlink.com

\begin{abstract}
Chimpanzees provide help to unrelated individuals in a broad range of situations. The pattern of helping within pairs suggests that contingent reciprocity may have been an important mechanism in the evolution of altruism in chimpanzees. However, correlational analyses of the cumulative pattern of interactions over time do not demonstrate that helping is contingent upon previous acts of altruism, as required by the theory of reciprocal altruism. Experimental studies provide a controlled approach to examine the importance of contingency in helping interactions. In this study, we evaluated whether chimpanzees would be more likely to provide food to a social partner from their home group if their partner had previously provided food for them. The chimpanzees manipulated a barpull apparatus in which actors could deliver rewards either to themselves and their partners or only to themselves. Our
\end{abstract}

\section{S. F. Brosnan ( $\square)$}

Department of Psychology, Georgia State University, PO Box 5010, Atlanta, GA 30302-5010, USA

e-mail: sbrosnan@gsu.edu

S. F. Brosnan · M. C. Mareno $\cdot$ S. P. Lambeth $\cdot$ S. J. Schapiro Michale E. Keeling Center for Comparative Medicine and Research, The University of Texas M. D. Anderson Cancer Center, Houston, TX, USA

J. B. Silk

Department of Anthropology, UCLA, Los Angeles, CA, USA

\section{J. Henrich}

Department of Psychology,

University of British Colombia, Vancouver, BC, Canada

J. Henrich

Department of Economics,

University of British Colombia, Vancouver, BC, Canada findings indicate that the chimpanzees' responses were not consistently influenced by the behavior of their partners in previous rounds. Only one of the 11 dyads that we tested demonstrated positive reciprocity. We conclude that contingent reciprocity does not spontaneously arise in experimental settings, despite the fact that patterns of behavior in the field indicate that individuals cooperate preferentially with reciprocating partners.

Keywords Chimpanzee $\cdot$ Pan troglodytes $\cdot$ Reciprocity · Prosocial behavior $\cdot$ Other-regarding behavior

\section{Introduction}

Both kin selection (Hamilton 1964) and contingent reciprocity (Axelrod and Hamilton 1981; Trivers 1971) may contribute to the evolution of altruism. While there is good evidence for kin biases in various forms of altruistic behavior across the animal kingdom (Dugatkin 1997), there is considerable controversy regarding the role of contingent reciprocity in sustaining altruism (Hammerstein 2003; Silk 2008). In order to demonstrate that contingent reciprocity is operating, it is necessary to show that the likelihood of providing help at one point of time is contingent on past interactions. It is difficult, if not impossible, to meet this burden of proof in naturalistic studies. This has led researchers to develop experimental protocols for studying reciprocity in a variety of species (Saguinus oedipus: Hauser et al. 2003; Gasterosteus aculeatus: Milinski 1987; Cyanocitta cristata: Stephens et al. 2002; Rattus norvegicus: Rutte and Taborsky 2007, 2008). Carefully designed experimental studies allow researchers to quantify pay offs, regulate opportunities for turn-taking, and monitor the sequence of interactions across time. Several experimental studies have 
demonstrated the presence of contingent reciprocity (Rutte and Taborsky 2008; Hauser et al. 2003), and there is some experimental evidence for contingent cooperation in chimpanzees (de Waal 1997; Melis et al. 2008). Here, we examine whether the opportunity for turn-taking in an experimental task increases the frequency of helpful (prosocial) behavior within familiar pairs of unrelated chimpanzees, Pan troglodytes. In this experiment, chimpanzees had the opportunity to provide one reward to themselves and an identical reward to their partner (prosocial option), or to provide one reward to themselves and nothing to their partner (selfish option). Previous work indicated that chimpanzees do not discriminate between these two options when there is no opportunity for turn-taking (Jensen et al. 2006; Silk et al. 2005; Vonk et al. 2008). We predicted that the opportunity for turn-taking would enhance the probability of choosing the prosocial option, and that chimpanzees would be sensitive to the behavior of their partners in previous rounds.

Chimpanzees are likely candidates for the development of contingent behavioral strategies because they meet the cognitive conditions which have been proposed as necessary for contingent reciprocity to operate. Stevens and Hauser (2004) argue that, aside from the prerequisites outlined by Trivers (1971; recipient benefit $>$ donor cost, repeated interaction, cheater detection), there are three additional cognitive requirements for the development of contingency, namely the ability to overcome temporal discounting biases, the capacity for numerical discrimination, and the capacity for learning and memory. Experimental evidence indicates that chimpanzees meet these requirements (temporal discounting: Beran and Evans 2006; numerical discrimination: Hanus and Call 2007; memory/ learning: Matsuzawa 2001).

In the wild, chimpanzees perform a variety of cooperative behaviors with unrelated partners, and the patterning of their interactions is consistent with predictions of the theory of reciprocal altruism. Male chimpanzees jointly patrol their territories, hunt collectively, share meat, groom one another, form coalitions in aggressive intragroup encounters, and cooperate in mate guarding (Goodall 1986; Muller and Mitani 2005). Grooming within dyads is more evenly balanced across bouts than within bouts (Gomes et al. 2008), suggesting that chimpanzees may keep track of their grooming interactions over considerable periods of time. While males show clear preferences for maternal kin (Langergraber et al. 2007), cooperation is also extended to unrelated males. In some populations, males more frequently provide these kinds of services to those individuals who are most likely to provide them with services in return (Duffy et al. 2007; Mitani et al. 2000; Watts 1998, 2002). Nonetheless, the observational data are correlational in nature and so do not demonstrate that cooperation is contingent on previous interactions. Thus, the role of contingent reciprocity in shaping these interactions remains unclear.

Limited experimental evidence suggests that contingent reciprocity may play some role in the patterning of food sharing in chimpanzees. For example, de Waal (1997) found that chimpanzees who gained possession of a bundle of leafy branches were more tolerant of transfers to individuals who had groomed them earlier in the day than they were of individuals who had not groomed them earlier. However, the absolute magnitude of this effect was relatively small, and grooming had more pronounced effects on food transfers among pairs of chimpanzees that did not frequently groom one another than among frequent grooming partners. While these data suggest that immediate contingencies may influence behavior in some circumstances, such contingencies may not be the only factor shaping tolerant responses in chimpanzees in this context. More recent studies indicate that chimpanzees show a weak tendency to help those who have previously helped them, but subjects did not assist this helpful individual more than another chimpanzee who had failed to help them (Melis et al. 2008). In another study, chimpanzees were capable of contingent reciprocity in a restricted paradigm, but not when the behavior must arise spontaneously (Yamamoto and Tanaka 2009).

We examined the potential for contingent reciprocity in captive chimpanzees in a simple barpull task. We chose the barpull apparatus for two reasons. First, these chimpanzees had completed two previous experiments using the same device, and were familiar with how it worked (Silk et al. 2005; Vonk et al. 2008). In addition, we would be able to directly compare our results across studies. Similar types of barpull devices have been used successfully with chimpanzees (Crawford 1937; Melis et al. 2006a, b), capuchins (Brosnan et al. 2006; de Waal and Berger 2000; Mendres and de Waal 2000), and callithrichids (Cronin et al., 2005; Cronin and Snowdon 2008; Burkart et al. 2007), suggesting that the device is appropriate for a range of nonhuman primates. Finally, we chose a payoff structure that rewarded the actor as well as the recipient, to increase the likelihood of prosocial (if not altruistic) acts.

In this experiment, individuals were tested in pairs. In each trial, one member of the pair could choose between two options: (1) deliver a food reward to itself and nothing to its partner (the selfish option, hereafter) or (2) deliver identical food rewards to itself and its partner (prosocial option). On the next trial, the partner was offered the same set of choices; this procedure was alternated across 16 trials per session (giving each chimpanzee eight possible pulls). Based on previous experiments using the same apparatus and the same set of options (Silk et al. 2005), we predicted that the chimpanzees would choose the prosocial option about half of the time on the first trial within each session. 
If chimpanzees are sensitive to the behavior of their partners (which they are when interacting with humans; Subiaul et al. 2007), then they are expected to preferentially choose the prosocial option when their partner had chosen the prosocial option on previous rounds and to preferentially chose the selfish option if their partner had not chosen the prosocial option on previous trials. If chimpanzees are insensitive to contingency in this context, then they will be unaffected by their partners' choice in previous trials.

\section{Methods}

Subjects

Chimpanzee subjects were drawn from six corral-housed groups at the Michale E. Keeling Center for Comparative Medicine and Research of the UT M. D. Anderson Cancer Center in Bastrop, TX. Each of these multi-male, multifemale groups of 7-15 individuals have been maintained as stable social units for up to 30 years. All groups have approximately the same age distribution among adults, although the number of immatures varies by group. These groups are housed in large outdoor enclosures with climbing structures and other forms of enrichment (Riddle et al. 1982), and also have access to an indoor area. They have ad libitum access to primate chow and water and receive at least four additional enrichment meals spread throughout the day.

Here we report the results of experiments involving 13 adult females who formed 11 different dyads. All animals were paired with members of their own social groups. When one chimpanzee was paired with more than one partner (range 1-3 partners per subject), all testing with one partner was completed before testing with another partner was initiated. Ten of these chimpanzees had been involved in two previous studies using the same apparatus (Silk et al. 2005; Vonk et al. 2008). Six of these chimpanzees had played the role of actor in previous experiments, and thus had had the opportunity to manipulate the apparatus, bringing rewards to themselves and/or their partners. Four had been potential recipients in previous experiments. These individuals had not had the opportunity to manipulate the barpull apparatus, but had watched other chimpanzees use the apparatus and had received food rewards from the apparatus.

\section{Experimental setup}

Chimpanzees were tested in indoor sections of their home enclosure. The chimpanzees were positioned in adjacent enclosures, divided by a wire mesh fence. They were able to see each other, vocalize, and do limited grooming

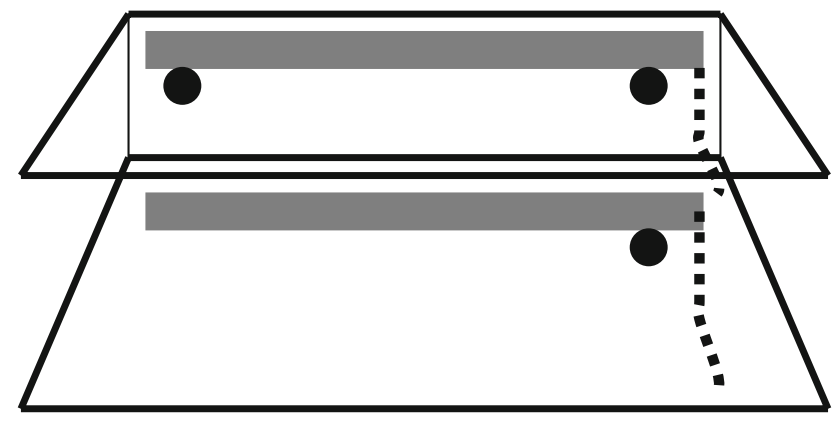

Recipient

Donor

Fig. 1 A schematic of the barpull apparatus. Two lexan trays were positioned on top of the other, with a vertical separation of approximately $45 \mathrm{~cm}$. The actor could choose to pull, using a rope handle (thick dotted lines) either of the two barpulls (gray bars) forward to receive food (black circles). The recipient only received food if the actor pulled the level baited on the recipient's side. The position of the ropes alternated from trial to trial, so each individual had the opportunity to pull on alternate trials. The actor and recipient were next to each other, separated by a mesh partition (thin dotted line). Here, the donor is on the right side of the mesh partition, the prosocial option is provided on the top level and the selfish option is provided on the bottom level

through this mesh barrier. The experimental apparatus was a two-tiered barpull apparatus (Fig. 1). One individual (the 'actor') could use this apparatus to choose one of two options (described below). In the next trial, the apparatus was adjusted so that the chimpanzee on the other side (the 'recipient') had an opportunity to operate the barpull for the next trial. The chimpanzees did not switch sides between trials within a session or between sessions. Below, we refer to the individual who was able to dispense food rewards on the current trial as the actor, and to the other individual as the recipient.

The barpull apparatus, consisting of two trays stacked vertically, spanned most of the width of the adjoining enclosures in which subjects were tested. The two trays, made of clear Lexan (plastic), were approximately $45 \mathrm{~cm}$ apart. Each tray had a colored metal sweeper mounted on the tray with tracks. Each sweeper had a rope handle (in the same color as the bar) attached to it on both sides. The ropes could be pulled back so that they were available on only one side; in this way we were able to alternate chimpanzees' ability to manipulate the barpull between trials. When one of these ropes was pulled, the sweeper that the rope was attached to moved along the tracks toward the chimpanzees. These two sweepers were connected with a mechanical apparatus so that when one sweeper was pulled forward, the other sweeper locked and the rope handle retracted several inches so that the chimpanzee could not reach it. Food placed on the Lexan tray in front of the sweeper was swept close to the 
edge of the enclosures when the handle was pulled, allowing the chimpanzees to obtain rewards.

Food rewards were chosen to maximize motivation of the subjects to attend to the study. Rewards for both subject and partner consisted of small, "bite-sized" chocolate, caramel and peanut candies that were approximately $16 \mathrm{~cm}^{3}$ in volume. These rewards are highly preferred by all subjects and are never received in the typical diet.

Subjects who had not been exposed to the barpull before were trained using the same series of training conditions that had been used in earlier studies. Training was designed to ensure that subjects understood that they could choose one of the two levels in each trial, without biasing them towards pulling one level over the other. In order to facilitate understanding of how the apparatus worked, chimpanzees viewed partners receiving rewards as a byproduct of their actions to obtain their own rewards during training, but were never encouraged to choose a particular option $(1 / 1$ or $1 / 0)$ or to choose the option which rewarded the partner.

Training sessions consisted of 2060 -s trials in which experimenter baited the trays (top first), then moved the apparatus within reach of the chimpanzees. The experimenter then left the room for the duration of the trial. If the actor pulled a handle within five trials, trials continued until the end of the session, or until the actor did not pull a handle for five consecutive trials. In this case, the next trial was a "demonstration" trial, in which the experimenter simultaneously pushed both sweepers all the way forward after baiting the tray. After the "demonstration" trial, there were five additional "delayed demonstration" trials in which the actor was given $30 \mathrm{~s}$ to pull a handle (while the experimenter was out of the room) before the experimenter pushed both trays forward. If at any point the actor pulled without prompting, unprompted trials began again. If this did not occur within five delayed demonstration trials, the actor was given two 90-s unprompted trials before resuming delayed demonstration trials, unless this would be the last pull of a session, in which case delayed demonstration trials continued. Unprompted trials served to keep the actor from just learning to wait for the experimenter to push the sweepers forward on each trial. Criterion required the actor to pull in eight of the last ten trials in a session without prompting. Following the procedures adopted in previous experiments (Silk et al. 2005; Vonk et al. 2008), individuals that did not reach criterion within five sessions were excluded from the experiment. All subjects repeated the training regardless of previous experience, to verify that they were still willing to utilize the apparatus.

\section{Testing}

Testing consisted of six 16-trial sessions per pair. Because subjects alternated roles, each chimpanzee controlled the apparatus for eight of these trials in each session (48 trials total). For each of these sessions, one subject played the role of actor in all odd trials and the other subject played the role of actor in all even trials; whether the chimpanzee pulled on odd or even trials alternated between sessions and it was randomly determined which subject pulled first in the first session. Trays were baited so that one tray had two identical rewards, the $1 / 1$ option (e.g. one reward for the actor and one for the recipient) and one tray, the 1/0 option, had only one reward, for the actor. Thus, the actor always received the same reward, regardless of her choice, but she could choose to reward her partner as well. All three rewards were identical, so the actor's choice did not affect the amount of food she received. The rewards were counterbalanced between top and bottom for each actor (so each actor had four trials per session in which the $1 / 1$ option was on top and four trials per session in which $1 / 1$ option was on the bottom). The order was determined randomly, except that no actor had more than three trials in a row that were baited in the same way. Trials in which the actor made no response were not re-run.

For each trial, the experimenter held up the rewards to be placed on the top tray, calling both subjects by name (to verify that they saw the rewards), then placed these rewards on the tray. If the option included two rewards, both rewards were placed simultaneously, one in each hand. This was then repeated for the bottom tray. After both trays were baited, the apparatus was pushed forward approximately $15 \mathrm{~cm}$ so that it could be accessed by the actor. Immediately after this, the experimenter left the room for $45 \mathrm{~s}$ to reduce the possibility that the experimenter was influencing behavior. A video camera recorded all trials. When the experimenter re-entered the room, she pulled back the apparatus, removed any rewards from the apparatus, switched the sides of the rope handles (so that the other chimpanzee could now pull) and began timing the next trial. Approximately 2 min separated each trial.

Each pair participated in only one session per day, and no pair was tested more than five times per week (typically pairs received 2-3 sessions per week). No subject participated in more than one pairing at a time. Testing sessions were conducted across a 12-month period. All trials were videotaped using a Canon digital video camera.

\section{Control condition}

After the completion of testing, each subject also completed a control to verify that they understood that the food that they could not reach was available to their partner on the other side of the partition. We did not run this test earlier in the experiment to avoid biasing the chimpanzees to pull the option which rewarded their partner, and to avoid possible confusion which could arise if they could reach food in 
the other enclosure during some trials or sessions, but not others.

For this, subjects were tested individually for 16 trials in a single session. The door between the two compartments was left open, allowing the subject full access to both sides of the testing area. Each tray was baited so that one food item (the same bite-sized candy bar) was placed on one level of one side of the barpull apparatus, and the ropes to pull in the trays were available on the other side. Thus, in order to get food, the subject had to pull the tray in on one side and then cross to the other side to obtain the food. This methodology was chosen to avoid presenting the chimpanzees with too many candy bars in a single session (a prohibition based on husbandry and veterinary concerns). The side on which the subject pulled (and food was available) alternated between trials within the session, so that the subject was able to pull on each side eight times. This was done to replicate the method used during the regular test.

If subjects understood the way that the apparatus worked, they were expected to pull in the tray and then walk through the door to obtain their reward from the other side. Only seven subjects were available for this control test, but all seven obtained rewards from the other side in at least one trial. Consistent with their behavior in the experimental trials, subjects pulled on $64 \%$ of all control trials. Subjects obtained rewards on 58\% of trials in which they made a response (range 100-50\%). One subject obtained rewards on every trial. For the six remaining subjects, subjects showed a strong preference for choosing their preferred level (upper/lower), even though it did not provide rewards. This was the source of all errors for four subjects; $71 \%$ of the errors for one subject; and $60 \%$ of the errors for another subject. All but one of these subjects overcame their bias at least once and obtained rewards by choosing their less preferred level.

\section{Data coding}

The experimenter (M.C.M.) coded the data during testing. One rater who did not participate in the experiment also coded $20 \%$ of the trials from videotape independently. The rater coded which handle the chimpanzee chose to pull. The rater agreed about which handle the actor pulled on 205 of 215 trials, yielding a Cohen's Kappa of 0.93 .

We did not code the behavior of recipients during testing because the video footage did not provide a good view of the chimpanzees' behavior inside the enclosures. There were no lights inside the dens (to protect the chimpanzees), the walls of the dens were painted gray, and the front of the dens was barred, so the footage of the interior portions of the dens was poor. The video cameras were set up to provide a clear image of the barpull apparatus, which was positioned outside the dens in a well lit area. Furthermore, to avoid biasing subjects, the experimenter left the room during testing, so live coding was not possible.

\section{Data analysis}

We used two complementary methods to analyze these data. First, in aggregate analyses, we calculated a series of conditional probabilities to compare the likelihood of a subject pulling the prosocial option on a trial given that her partner pulled the prosocial option or the selfish option on previous trials. Second, to analyze whether the actor's behavior was influenced by the previous behavior of her partner, we used a logistic regression model using clustered robust standard errors to deal with the non-independence of repeated observations from the same dyad. The actor's choice (prosocial or selfish) was the dependent variable. The independent variables included the outcome in previous trials in which the partner pulled (prosocial, selfish, no response), trial number (1-16), session number (1-6), and position of the prosocial option (upper or lower). Because the actors might have attended to their partners' behavior across multiple trials, we examined the effect of behavior (a) in the previous trial, (b) the previous two trials, and (c) the previous three trials, as has been done in previous studies (e.g. Chen and Hauser 2005). In addition, because of the dependencies in the data due to individuals participating in multiple dyads, we examined the effect of behavior on the previous trial(s) for each dyad in separate regressions.

In some trials, actors made no response. In the analyses reported below, we treated these trials the same as selfish responses because in both cases the actor did not deliver a food reward to the recipient. To make sure that this decision did not influence the results, we also conducted multinomial regression analyses in which all three choices (prosocial, selfish, and no response) by actors and partners on previous trials were categorized separately. None of the results reported below were substantially altered (see Tables 1, 2).

\section{Results}

Subjects' behavior was largely unaffected by their partners' behavior in previous trials. There are four possible sequences of outcomes across a pair of successive trials: prosocial/prosocial, prosocial/selfish, selfish/prosocial, selfish/selfish. If the chimpanzees deployed contingent behavioral strategies, the prosocial/prosocial and selfish/selfish sequences should predominate. Figure 1a (see also Table 3) shows that actors were just as likely to choose the prosocial option given that their partner had chosen the selfish option or had made no response in the previous round $(45 \%)$ as 
Table 1 The distribution and conditional probability of actor's responses given behavior of partner on previous trial

\begin{tabular}{lllll}
\hline $\begin{array}{l}\text { Actor's } \\
\text { behavior }\end{array}$ & \multicolumn{2}{l}{ Partner's behavior in last trial } & Total \\
\cline { 2 - 4 } & $1 / 1$ & $1 / 0$ & No response & \\
\hline $1 / 1$ & 195 & 177 & 67 & 439 \\
& 0.45 & 0.43 & 0.48 & \\
$1 / 0$ & $(0.38-051)$ & $(0.33-0.52)$ & $(0.40-0.56)$ & \\
& 173 & 164 & 70 & 407 \\
& 0.40 & 0.40 & 0.50 & \\
No response & 68 & $(0.31-0.48)$ & $(0.41-0.59)$ & \\
& 0.16 & 0.18 & 2 & 144 \\
& $(0.04-0.27)$ & $(0.02-0.33)$ & $(0-0.039)$ & \\
Total & 436 & 415 & 139 & 990 \\
\hline
\end{tabular}

The parentheses contain $95 \%$ confidence intervals based on assuming independence only among dyads

Table 2 Multinomial regression analyses, including 1/1, 1/0, and no response as separate variables

$\begin{array}{lllll}\text { Odds } & 95 \% \text { Bounds } & \text { SE } & \text { z-Score } P \\ \text { ratio } & \text { Upper Lower } & & & \end{array}$

Effect of pulling 1/1 (relative to 1/0)

\begin{tabular}{lllllll} 
Partner's pull & 0.97 & 0.81 & 1.15 & 0.09 & -0.37 & 0.071 \\
Session & 0.097 & 0.089 & 1.07 & 0.0 .5 & -0.61 & 0.54 \\
Trial & 1.01 & 0.097 & 1.05 & 0.02 & 0.047 & 0.064 \\
Baiting & 2.44 & 0.92 & 6.45 & 1.21 & 1080 & 0.07 \\
Effect of pulling nothing & (relative to 1/0) & & & \\
Partner's pull & 1.32 & 0.99 & 1.78 & 0.20 & 1.89 & 0.058 \\
Session & 0.90 & 0.080 & 1.02 & 0.0 .6 & -1.61 & 0.11 \\
Trial & 1.05 & 1.02 & 1.09 & 0.02 & 2.8 & 0.005 \\
Baiting & 1.90 & 1.14 & 3.17 & 0.20 & 1.85 & 0.06 \\
\hline
\end{tabular}

This measures the cumulative effect of partner's behavior in last three trials on actor's behavior. Odds ratios are calculated with respect to pulling $1 / 0$

Results indicate that subjects are marginally (but not significantly) more likely to do nothing if they recently got food from the donor $(P=0.058$, bottom table). Baiting remains marginally significant. Wald $\chi^{2}=36.82, P<0.001$

they were to choose the prosocial option given that their partner had also chosen the prosocial option (44\%). Similarly, the likelihood of choosing the selfish option or making no response was not influenced by what their partner had done in the previous trial. Analyses based on behavior across the previous two trials and the previous three trials provide very similar results (Fig. 2b, c; see also Tables 4, 5).

The logistic regression analyses confirm that the chimpanzees did not respond to the behavior of their partners in previous trials in a consistent way (Table 6). We examined the effects of trial number, baiting position, partners' past
Table 3 The distribution and conditional probability of actor's responses behavior of partner on previous trial

\begin{tabular}{llll}
\hline Actor's behavior & \multicolumn{2}{l}{ Partner's behavior in last trial } & Total \\
\cline { 2 - 3 } & $1 / 1$ & $1 / 0$ or NR & \\
\hline $1 / 1$ & 195 & 244 & 439 \\
& 0.45 & 0.44 & \\
1/0, No response & $(0.38-0.51)$ & $(0.37-0.51)$ & \\
& 241 & 310 & 551 \\
Total & 0.55 & 0.56 & \\
\hline
\end{tabular}

The parentheses contain $95 \%$ confidence intervals based on assuming independence only among dyads

choice(s), and session number on the likelihood of choosing the prosocial option. The odds ratio (e.g. the odds of a given choice based on the partner's previous choices) for partners past choice(s) on Table 6 are always close to one and never distinguishable from zero. In fact, the odds ratios indicate that the chimpanzees were much more strongly affected by the position of the prosocial option (upper/ lower) than by whether their partners had chosen the prosocial option in previous trial(s). There was no significant effect of session or trial on the chimpanzees' responses, indicating that the chimpanzees did not become more responsive to their partner's behavior as the experiment progressed (Fig. 3).

We also conducted separate logistic regression analyses for each dyad. The likelihood of choosing the prosocial option was not significantly affected by the behavior of the partner in the previous round for any of the dyads. The $P$ values on the odds ratio for partner's previous choice was always greater than 0.19 for all 11 runs. However, when we considered behavior in the previous two trials, evidence of contingency emerged for one dyad, and when we included behavior in the previous three trials a different dyad showed evidence of contingency. In one dyad, the females were more likely to choose the prosocial option as the number of prosocial choices by their partners in previous rounds increased; in the other dyad, the pattern was reversed. Note that a $P$ value of 0.05 indicates that for every 20 tests, one type I (false positive) error is expected to occur by chance. Thus, with 33 tests, approximately 1.7 significant results are expected to arise by chance.

\section{Discussion}

The chimpanzees that we tested did not respond to the behavior of their partners in a contingent manner. As a group, they were no more likely to choose the prosocial option if their partner had chosen the prosocial option in the 

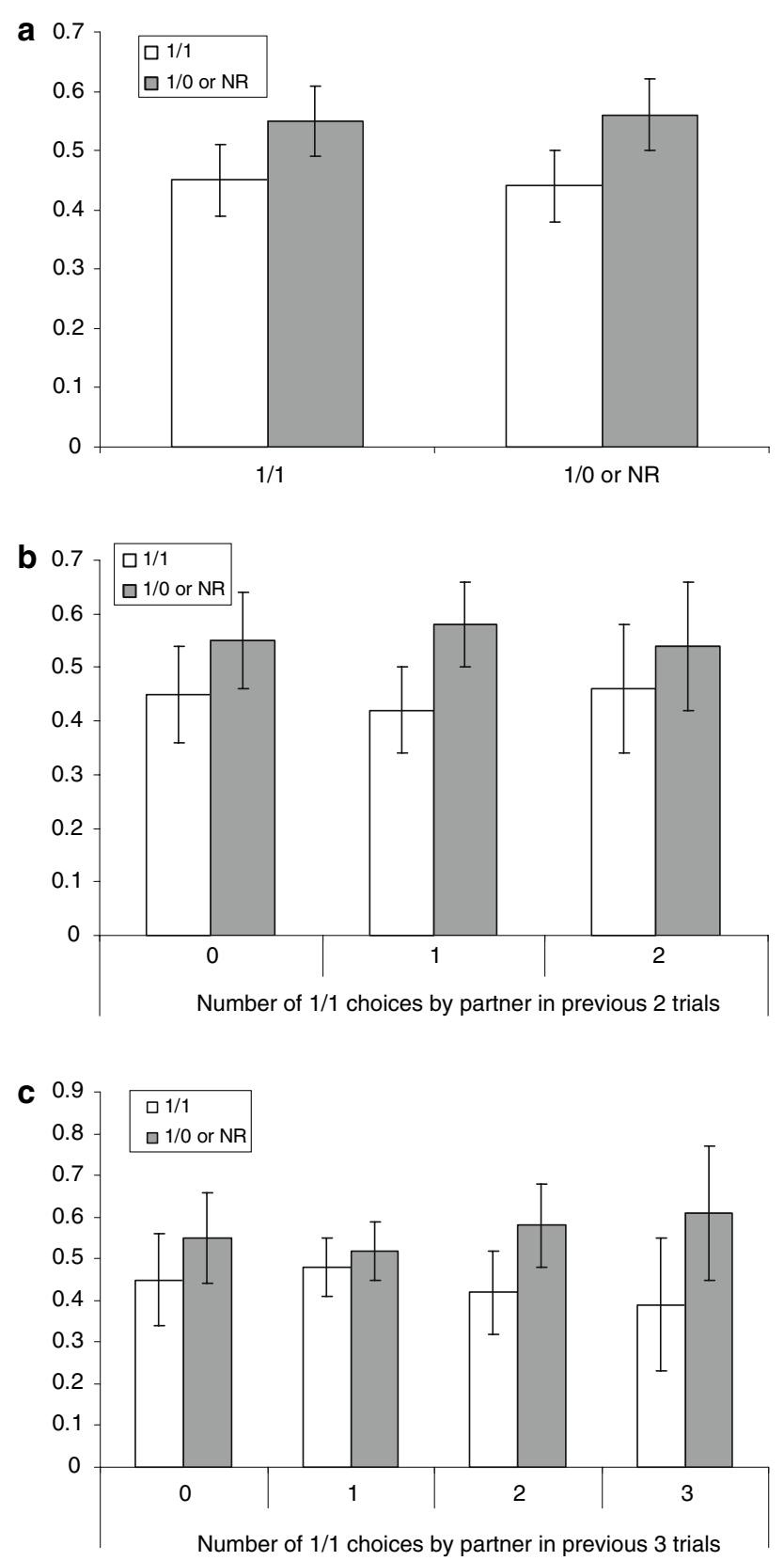

Fig. 2 The distribution and conditional probability of actor's responses given a behavior of partner on previous trial, $\mathbf{b}$ number of $1 / 1$ choices made by partner in previous two trials, $\mathbf{c}$ number of $1 / 1$ choices made by partner in previous three trials. The bars indicate $95 \%$ confidence intervals based on assuming independence only among dyads

previous trial than if their partner had chosen the selfish option or done nothing. The results obtained from the aggregate data generally correspond to the results of analyses on individual pairs. We found evidence of contingent responses in two of the 13 dyads that we tested; in one pair, prosocial choices in the previous two rounds increased the chance of prosocial choices in the current round, but in the other pair of females, prosocial choices in the last three rounds decreased the likelihood of prosocial choices in the
Table 4 The distribution and conditional probability of actor's responses number of $1 / 1$ choices made by partner in previous two trials

\begin{tabular}{lllll}
\hline $\begin{array}{l}\text { Actor's } \\
\text { behavior }\end{array}$ & \multicolumn{2}{l}{$\begin{array}{l}\text { Number of 1/1 choices } \\
\text { by partner in last two trials }\end{array}$} & Total \\
\cline { 2 - 4 } & 0 & 1 & 2 & \\
\hline $1 / 1$ & 123 & 178 & 76 & 377 \\
& 0.45 & 0.42 & 0.46 & \\
$1 / 0$, & $(0.36-0.54)$ & $(0.35-0.50)$ & $(0.34-0.58)$ & \\
no response & 149 & 243 & 89 & 481 \\
& 0.55 & 0.58 & 0.54 & \\
Total & $(0.49-0.61)$ & $(0.53-0.63)$ & $(0.46-0.62)$ & \\
\hline
\end{tabular}

The parentheses contain $95 \%$ confidence intervals based on assuming independence only among dyads

Table 5 The distribution and conditional probability of actor's responses number of $1 / 1$ choices made by partner in previous three trials

\begin{tabular}{llllll}
\hline $\begin{array}{l}\text { Actor's } \\
\text { behavior }\end{array}$ & \multicolumn{2}{l}{$\begin{array}{l}\text { Number of 1/1 choices } \\
\text { by partner in last three trials }\end{array}$} & Total \\
\cline { 2 - 5 } & 0 & 1 & 2 & 3 & \\
\hline $1 / 1$ & 62 & 132 & 108 & 23 & 377 \\
& 0.45 & 0.48 & 0.42 & 0.39 & \\
& $(0.35-0.56)$ & $(0.41-0.55)$ & $(0.32-0.52)$ & $(0.22-0.55)$ \\
$1 / 0$, no & 75 & 143 & 147 & 36 & 481 \\
response & 0.55 & 0.52 & 0.58 & 0.61 & \\
& $(0.46-0.63)$ & $(0.46-0.58)$ & $(0.51-0.64)$ & $(0.47-0.74)$ \\
Total & 137 & 275 & 255 & 59 & 858 \\
\hline
\end{tabular}

The parentheses contain $95 \%$ confidence intervals based on assuming independence only among dyads

current round. The opportunity for turn-taking in this experiment did not elevate the likelihood of choosing the prosocial option over the level observed in previous experiments in which roles of actor and recipient did not alternate. In the present experiment, actors who made a response chose the prosocial option $52 \%$ of the time $(95 \%$ CI $48-56 \% ; n=905$ trials in 11 dyads), which is not distinguishable from the level observed in a previous study in which the chimpanzees did not alternate roles (49\%; 95\% CI 45-5\%; $n=554$ trials in 11 independent dyads; Silk et al. 2005). Moreover, in the present study there was no effect of trial or session. This indicates that the chimpanzees did not develop contingent strategies over the course of the experiment.

The absence of contingent responses in this experiment is notable because it would have provided a mechanism for individuals to increase the likelihood of obtaining rare and highly valued rewards for themselves. If individuals rewarded their partners for prosocial choices by making prosocial choices themselves, they might have been able to shape their partners' behavior and increase the number of rewards that they obtained themselves in the course of the experiment. Moreover, their motivation to adopt this 
Table 6 Logistic regression analyses

${ }^{a}$ Wald $\chi^{2}=5.95, P=0.20$

b Wald $\chi^{2}=3.91, P=0.42$

c Wald $\chi^{2}=4.50, P=0.34$

Fig. 3 The frequency of prosocial $(1 / 1)$ choices over the course of the six sessions. The dashed horizontal line at 0.5 indicates chance levels of choosing the prosocial option over the selfish (1/0) option. Note that $Y$-axis scale ranges only from 45 to $55 \%$

\begin{tabular}{llllll}
\hline Odds ratio & & $95 \%$ Bounds & S.E. & z-Score & $P$ \\
\cline { 2 - 3 } & & & & \\
& Upper & Lower & & & \\
\hline
\end{tabular}

(a) Effect of partner's behavior in last trial on actor's behavior ${ }^{\text {a }}$

$\begin{array}{llllrrr}\text { Partner chose 1/1 } & 1.01 & 0.85 & 1.20 & -0.09 & 0.15 & 0.88 \\ \text { Session } & 0.98 & 0.93 & 1.04 & 0.03 & -0.54 & 0.59 \\ \text { Trial } & 1.00 & 0.97 & 1.02 & 0.01 & -0.43 & 0.67 \\ \text { Baiting } & 2.07 & 0.97 & 4.42 & 0.80 & 1.88 & 0.06\end{array}$

(b) Effect of partner's behavior in last two trials on actor's behavior ${ }^{\text {b }}$

$\begin{array}{lllllrr}\text { Partner chose 1/1 } & 0.099 & 0.79 & 1.26 & 0.12 & -0.05 & 0.96 \\ \text { Session } & 0.99 & 0.93 & 1.07 & 0.04 & -0.17 & 0.86 \\ \text { Trial } & 1.00 & 0.97 & 1.04 & 0.02 & 0.26 & 0.80 \\ \text { Baiting } & 2.07 & 0.093 & 4.62 & 0.85 & 1.78 & 0.08\end{array}$

(c) Effect of partner's behavior in last three trials on actor's behavior ${ }^{\mathrm{c}}$

\begin{tabular}{lccccrr} 
Partner chose 1/1 & 0.89 & 0.75 & 1.05 & 0.08 & -1.28 & 0.17 \\
Session & 1.00 & 0.92 & 1.09 & 0.04 & 0.05 & 0.96 \\
Trial & .099 & 0.96 & 1.03 & 0.02 & -0.29 & 0.77 \\
Baiting & 2.02 & 0.87 & 4.73 & 0.88 & 1.63 & 0.10 \\
\hline
\end{tabular}

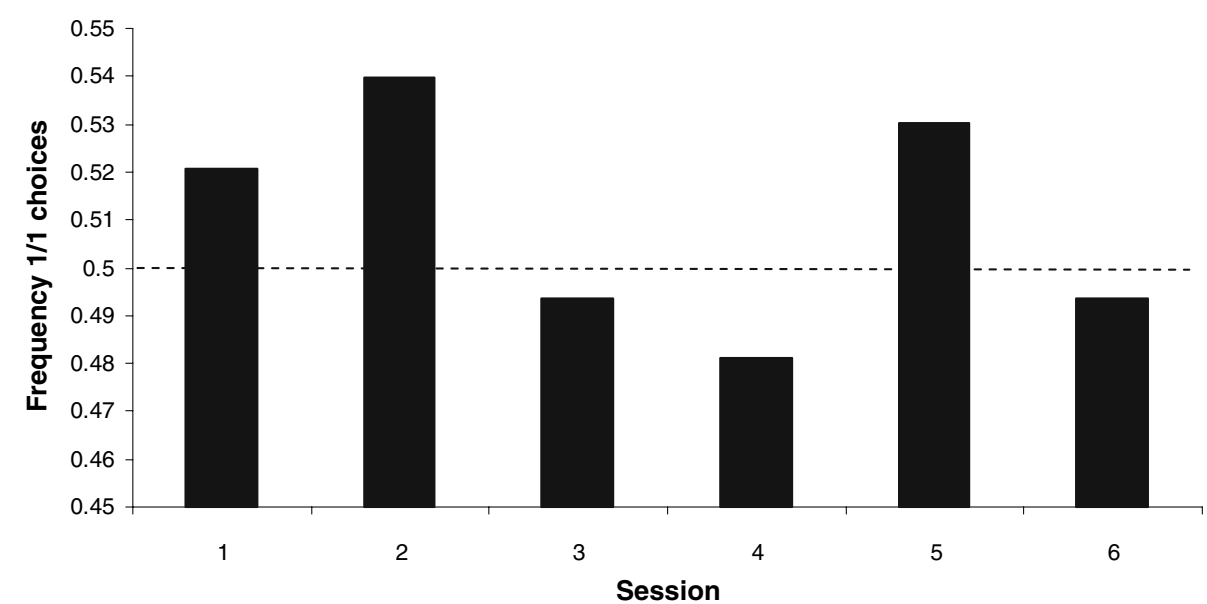

strategy would not have competed with selfish preferences because actors incurred no costs when they chose the prosocial option instead of the selfish option.

These results are striking because chimpanzees show considerable cognitive complexity (Matsuzawa 2001), exhibit strong preferences for cooperating with particular partners in the wild (Muller and Mitani 2005), seem to be able to keep track of exchanges across currencies (in the sense of scorekeeping: de Waal 1997; Duffy et al. 2007; Mitani et al. 2000; Watts 1998, 2002), and successfully collaborate on mutualistic tasks in the laboratory (Melis et al. 2006a, b; Crawford 1937, although see Tomasello and Call 1997). However, our results are compatible with recent work which suggests that contingent reciprocity is not a robust force in experimental settings. Melis et al. (2008) found that chimpanzees show slightly increased levels of helping behavior towards individuals who helped them in the past, but their willingness to assist cooperative partners did not differ from their willingness to assist uncooperative partners. Taken together, these studies suggest that chimpanzees, despite their cognitive sophistication, do not adopt contingent reciprocal strategies in these controlled experimental settings. Below we explore some possibilities for why this is the case.

One conclusion that could be drawn from these studies is that chimpanzees are not capable of deploying contingent reciprocal strategies, and other mechanisms underlie exchange in natural settings. Some of the possible alternative mechanisms would include attitudinal reciprocity (de Waal 2000) or biological market models (Noë and Hammerstein 1995; Noë et al. 2001; Barrett et al. 1999; de Waal 1997). Those who are skeptical of the evidence for reciprocity in nature are likely to favor this possibility. However, those who believe that the correlational evidence 
is suggestive of contingent reciprocity are likely to think that the experiments failed to elicit the kind of behavioral strategies that chimpanzees deploy in the wild (e.g. Hammerstein 2003). Below we discuss some of the possible reasons that experimental studies may fail to elicit contingent reciprocity.

As with any experiment, elements of the task may have affected the chimpanzees' performance (Noë 2006). The chimpanzees that we tested were not able to choose their own partners and could not control the timing of their interactions, as they can in more naturalistic settings. If relationship quality influences the propensity for contingent reciprocity, then restrictions on partner choice may have influenced the results that we obtained. However, de Waal (1997) found stronger evidence of contingency among individuals that did not groom often than frequent grooming partners, which suggests that strong bonds are not a prerequisite for the development of contingent altruism in chimpanzees. It is also possible that contingent reciprocity operates over a longer time frame than experimental studies consider. For example, Gomes et al. (2008) found that grooming was more evenly balanced over the course of a week than within days or within single bouts. Thus, it is possible that partner's behavior during a single experimental trial has less impact on the actor's behavior than the many social interactions that they have had in the past.

The lack of contingency observed in experimental settings might also be related to the fact that chimpanzees and other primates do not keep precise track of favors given and received. de Waal (2000) has proposed that reciprocity may be maintained by 'attitudinal reciprocity,' in which individuals' responses are based on the positive feelings generated when a partner gives a favor, not on an exact accounting of favors given and received. Recent studies suggest that the hormone oxytocin may provide a proximate mechanism that regulates such feelings. In humans, oxytocin increases trust and generosity in experimental settings, even when subjects interact with strangers (Kosfeld et al. 2005). In primates, oxytocin is released during grooming (Morhenn et al. 2008). One drawback to this potential mechanism is that it may be possible for individuals to benefit by shirking unless there is some mechanism that translates costs and benefits into feelings or affect (Silk 2005).

Chimpanzees may not develop contingent strategies in this task because their evolved psychology for altruism does not extend to food. Although chimpanzees are well known for permitting meat transfers in the wild (Mitani and Watts 2001; Nishida et al. 1992) and mothers sometimes allow their infants access to food scraps that they possess (Nishida and Taylor 1996; Silk 1979), there is some dispute about whether food exchanges among adults are better characterized as tolerated theft (e.g. Gilby 2006) or voluntary sharing (Hiraiwa-Hasegawa 1990; Mitani 2006).
Recent studies of food transfers between mothers and infants suggest that mothers are reluctant to give up preferred food items, and do not often initiate food transfers to their infants (Ueno and Matsuzawa 2004). This has led Warneken et al. (2007) to suggest that the use of food rewards in experimental studies like this one may obscure the propensity for helpful behavior because chimpanzees treat all interactions involving food as part of a zero-sum game. If this is the case, then self-interested preferences may overwhelm other motivations, including other-regarding preferences, when food rewards are salient (Warneken et al. 2007).

It also is possible that chimpanzees (and other primates) might practice reciprocal altruism in the wild, but do not display contingent responses in experimental settings because they do not have a domain-general capacity for contingent reciprocity. Chimpanzees in the wild selectively exchange meat with the same individuals that they hunt with, groom, and support in agonistic interactions (Mitani 2006), which suggests that wild chimpanzees may trade goods (food) for services in an exchange economy. The chimpanzees that we tested may not have consistently chosen the prosocial option after their partner chose the prosocial option (and vice versa) because they did not associate the task that they were confronted with in these experiments with the kinds of situations that elicit contingent reciprocal responses in more natural circumstances. This could explain why our results differ from the results obtained in more naturalistic controlled studies of contingent reciprocity in vervets (Seyfarth and Cheney 1984), macaques (Hemelrijk 1994), and chimpanzees (de Waal 1997). However, capuchins (de Waal and Berger 2000) and tamarins (Hauser et al. 2003) have been tested with devices very similar to the device used in this study, and produced more convincing evidence for contingent reciprocity than we observed in this study.

Much of the evidence for exchanges in the wild is based on services, such as grooming and support, rather than goods, such as food. Service exchanges and service economies may be more likely to develop than those involving goods because service economies avoid the issues of property, risk, and ownership inherent in goods (Brosnan et al. 2008). While it is possible for food items to be acquired and quickly exchanged, or consumed immediately and stored as fat on the recipient's body, the full benefit of exchanging goods requires extra-bodily storage, and risks theft, loss, or degradation. Services, on the other hand, do not require storage and typically cannot be taken by force. Beyond kinbased transfers (e.g., milk to offspring), it seems plausible that what evolved first was the capacity or motivation to exchange services, followed by interchange of goods for services and, ultimately, goods for goods (Brosnan et al. 2008). 
The findings presented here suggest that contingent reciprocity does not spontaneously arise in experimental settings, even when it would seem to be an advantageous strategy for individuals to adopt. Although chimpanzees seem to cooperative preferentially with reciprocating partners in the wild, the mechanisms underlying their exchanges remain uncertain. The apparent discontinuity between the patterning of altruistic behavior within dyads in the field and in the laboratory requires further investigation. More systematic efforts to document contingent behavioral strategies in naturalistic settings and more creative efforts to simulate ecologically relevant opportunities for contingent cooperation in the laboratory may help to resolve this paradox and shed light on the mechanisms that favor the evolution of cooperation in apes and other primates.

Acknowledgments This work was supported by grants from the MacArthur Foundation Preferences Network to J.B.S. and J.H. S.F.B. was funded in part by NSF grant SES 0729244 and NIH Institutional Research and Academic Career Development grant \#K12 GM0068005 to Emory University. We thank Megan Ahlgren and Marie Butcher for assistance with data collection and Patrick Dougall for assistance with coding and inter-observer reliability. Support for the Bastrop chimpanzee colony comes from NIH/NCRR U42-RR015090. UTMDACC is fully accredited by AAALAC. All experiments described in this paper comply with the laws of the country in which they were performed.

Open Access This article is distributed under the terms of the Creative Commons Attribution Noncommercial License which permits any noncommercial use, distribution, and reproduction in any medium, provided the original author(s) and source are credited.

\section{References}

Axelrod R, Hamilton WD (1981) The evolution of cooperation. Science 211:1390-1396

Barrett L, Henzi SP, Weingrill T, Lycett JE, Hill RA (1999) Market forces predict grooming reciprocity in female baboons. Proc $\mathrm{R}$ Soc Lond Ser B 266:665-670

Beran MJ, Evans TA (2006) Maintenance of delay of gratification by four chimpanzees (Pan troglodytes): the effects of delayed reward visibility, experimenter presence, and extended delay intervals. Behav Process 73:315-324

Brosnan SF, Freeman C, de Waal FBM (2006) Partner's behavior, not reward distribution, determines success in an unequal cooperative task in capuchin monkeys. Am J Primatol 68:713-724

Brosnan SF, Grady M, Lambeth S, Schapiro S, Beran MJ (2008) Chimpanzee autarky. PLoS ONE 3(1):e1518

Burkart J, Fehr E, Efferson C, van Schaik CP (2007) Other-regarding preferences in a non-human primate: common marmosets provision food altruistically. Proc Natl Acad Sci 104(50):19762-19766

Chen K, Hauser M (2005) Modeling reciprocation and cooperation in primates: evidence for a punishing strategy. J Theor Biol 235:5-12

Crawford M (1937) The cooperative solving of problems by young chimpanzees. Comp Psychol Monogr 14(2):1-88

Cronin KA, Snowdon CT (2008) The effects of unequal reward distributions on cooperative problem solving by cotton-top tamarins (Saguinus oedipus). Anim Behav 75:245-257
Cronin KA, Kurian AV, Snowdon CT (2005) Cooperative problem solving in a cooperatively breeding primate. Anim Behav 69(1): 133-142

de Waal FBM (1997) The chimpanzee's service economy: food for grooming. Evol Human Behav 18:375-386

de Waal FBM (2000) Attitudinal reciprocity in food sharing among brown capuchin monkeys. Anim Behav 60:253-261

de Waal FBM, Berger ML (2000) Payment for labour in monkeys. Nature 404:563

Duffy KG, Wrangham RW, Silk JB (2007) Male chimpanzees exchange political support for mating opportunities. Curr Biol 17(15):R586

Dugatkin LA (1997) Cooperation among animals: an evolutionary perspective. Oxford University Press, New York

Gilby IC (2006) Meat sharing among the Gombe chimpanzees: harassment and reciprocal exchange. Anim Behav 71:953-963

Gomes CM, Mundry R, Boesch C (2008) Long-term reciprocation of grooming in wild West African chimpanzees. Proc R Soc Lond B. doi:10.1098/rspb.2008.1324

Goodall J (1986) The chimpanzees of Gombe. The Belknap Press of Harvard University Press, Cambridge

Hamilton WD (1964) The genetical evolution of social behavior. I. J Theor Biol 7:1-16

Hammerstein P (2003) Why is reciprocity so rare in social animals? In: Hammerstein P (ed) Genetic and cultural evolution of cooperation. MIT Press, Cambridge, pp 84-93

Hanus D, Call J (2007) Discrete quantity judgments in the great apes (Pan paniscus, Pan troglodytes, Gorilla gorilla, Pongo pygmaeus): the effect of presenting whole sets versus item-by-item. J Comp Psychol 121:241-249

Hauser MD, Chen MK, Chen F, Chuang E (2003) Give unto others: genetically unrelated cotton-top tamarin monkeys preferentially give food to those who altruistically give food back. Proc R Soc Lond B 270:2363-2509

Hemelrijk CK (1994) Support for being groomed in long-tailed macaques, Macaca fascicularis. Anim Behav 48:479-481

Hiraiwa-Hasegawa M (1990) The role of food sharing between mother and infant in the ontogeny of feeding behavior. In: Nishida $\mathrm{T}$ (ed) The chimpanzees of Mahale mountains. Tokyo University Press, Tokyo, pp 267-275

Jensen K, Hare B, Call J, Tomasello M (2006) What's in it for me? Self-regard precludes altruism and spite in chimpanzees. Proc R Soc Lond B 273:1013-1021

Kosfeld M, Heinrichs M, Zak PJ, Fischbacher U, Fehr E (2005) Oxytocin increases trust in humans. Nature 435:673-676

Langergraber KE, Mitani JC, Vigilant L (2007) The limited impact of kinship on cooperation in wild chimpanzees. Proc Natl Acad Sci 104:7786-7790

Matsuzawa T (ed) (2001) Primate origins of human cognition and behavior. Springer, Tokyo

Melis AP, Hare B, Tomasello M (2006a) Chimpanzees recruit the best collaborators. Science 311:1297-1300

Melis AP, Hare B, Tomasello M (2006b) Engineering cooperation in chimpanzees: tolerance constraints on cooperation. Anim Behav $72: 275-286$

Melis AP, Hare B, Tomasello M (2008) Do chimpanzees reciprocate favours? Anim Behav 76:951-962

Mendres KA, de Waal FBM (2000) Capuchins do cooperate: the advantage of an intuitive task. Anim Behav 60(4):523-529

Milinski M (1987) Tit for tat in sticklebacks and the evolution of cooperation. Nature 325:433-435

Mitani JC (2006) Reciprocal exchange in chimpanzees and other primates. In: Kappeler P, van Schaik CP (eds) Cooperation in primates and humans: evolution and mechanisms. Springer, Berlin, pp 101-113

Mitani JC, Watts DP (2001) Why do chimpanzees hunt and share meat? Anim Behav 61(5):915-924 
Mitani JC, Merriwether DA, Zhang C (2000) Male affiliation, cooperation and kinship in wild chimpanzees. Anim Behav 59(4):885-893

Morhenn VB, Park JW, Piper E, Zak PJ (2008) Monetary sacrifice among strangers is mediated by endogenous oxytocin release after physical contact. Evol Human Behav 29:375-383

Muller M, Mitani JC (2005) Conflict and cooperation in wild chimpanzees. In: Slater PJB, Rosenblatt J, Snowdon C, Roper T, Naguib $M$ (eds) Advances in the study of behavior. Elsevier, New York, pp 275-331

Nishida T, Taylor L (1996) Food transfer between mother and infant chimpanzees of the Mahale Mountains National Park, Tanzania. Int J Primatol 17:947-968

Nishida T, Hasegawa T, Hayaki H, Takahata Y, Uehara S (1992) Meatsharing as a coalition strategy by an alpha male chimpanzee? In: Nishida T, McGrew WC, Marler P, Pickford M, de Waal FBM (eds) Topics in primatology: human origins, vol 1. University of Tokyo Press, Tokyo, pp 159-174

Noë R (2006) Cooperation experiments: coordination through communication versus acting apart together. Anim Behav 71:1-18

Noë R, Hammerstein P (1995) Biological markets. Trends Ecol Evol 10:336-339

Noë R, van Hooff JARAM, Hammerstein P (2001) Economics in nature: social dilemmas, mate choice, and biological markets. Cambridge University Press, Cambridge

Riddle KE, Keeling ME, Alford PL, Beck TF (1982) Chimpanzee holding, rehabilitation and breeding: facilities design and colony management. Lab Anim Sci 32:525-533

Rutte C, Taborsky M (2007) Generalized reciprocity in rats. PLoS Biol 5:e196

Rutte C, Taborsky M (2008) The influence of social experience on cooperative behavior of rats (Rattus norvegicus): direct versus generalized reciprocity. Behav Ecol Sociobiol 62:499-505

Seyfarth RM, Cheney DL (1984) Grooming, alliances, and reciprocal altruism in vervet monkeys. Nature 308:541-543

Silk JB (1979) Feeding, foraging and food sharing behavior of immature chimpanzees. Folia Primatol 31:123-142

Silk JB (2005) The evolution of cooperation in primate groups. In: Gintis H, Bowles S, Boyd R, Fehr E (eds) Moral sentiments and material interests: on the foundations of cooperation in economic life. MIT Press, Cambridge, pp 43-73

Silk JB (2008) The strategic dynamics of cooperation in primate groups. Adv Study Behav 37:1-42

Silk JB, Brosnan SF, Vonk J, Henrich J, Povinelli DJ, Richardson AS et al (2005) Chimpanzees are indifferent to the welfare of unrelated group members. Nature 437:1357-1359

Stephens DW, McLinn CM, Stevens JR (2002) Discounting and reciprocity in an interacted prisoner's dilemma. Science 298:2216-2218

Stevens JR, Hauser MD (2004) Why be nice? Psychological constraints on the evolution of cooperation. Trends Cogn Sci 8:60-65

Subiaul F, Vonk J, Okamoto-Barth S, Barth J (2007) Do chimpanzees learn reputation by observation? Evidence from direct and indirect experience with generous and selfish strangers. Anim Cogn 11:611-623

Tomasello M, Call J (1997) Primate cognition. Oxford University Press, Oxford

Trivers RL (1971) The evolution of reciprocal altruism. Q Rev Biol 46:35-57

Ueno A, Matsuzawa T (2004) Food transfer between chimpanzee mothers and their infants. Primates 45:231-239

Vonk J, Brosnan SF, Silk JB, Henrich J, Richardson AS, Lambeth S et al (2008) Chimpanzees do not take advantage of very low cost opportunities to deliver food to unrelated group members. Anim Behav 75:1757-1770

Warneken F, Hare B, Melis AP, Hanus D, Tomasello M (2007) Spontaneous altruism by chimpanzees and young children. PLoS Biol $5(7): \mathrm{e} 184$

Watts DP (1998) Coalitionary mate guarding by male chimpanzees at Ngogo, Kibale National Park, Uganda. Behav Ecol Sociobiol $44: 43-55$

Watts DP (2002) Reciprocity and interchange in the social relationships of wild male chimpanzees. Behaviour 139:343-370

Yamamoto S, Tanaka M (2009) Do chimpanzees (Pan troglodytes) spontaneously take turns in a reciprocal cooperation task? J Comp Psychol (in press) 